\title{
Characteristics of Object-oriented Soft Concepts in a Soft Context
}

\author{
Won Keun Min \\ Department of Mathematics, Kangwon National University, Chuncheon, 24341, Korea. \\ ORCID: 0000-0002-3439-2255.
}

\begin{abstract}
We introduced a new type of soft concept called object oriented soft concept(simply, $m$-concept) based on soft sets, which is independent of the notion of soft concepts in a soft context. The purpose of this work is to study the topological structure in the collection of all the object oriented soft concepts in a soft context. We show that the collection of all the object oriented soft concepts in a soft context is a supratopology. Moreover, we introduce the notions of independent $m$-concept(object oriented soft concept) and dependent $m$-concept in a soft context. Using the notions, we show that the set of all independent $m$-concepts completely determines every $m$-concept in a given soft context.
\end{abstract}

Key words and phrases: Formal concepts, soft concepts, object oriented soft concepts, independent $m$-concepts

1991 Mathematics Subject Classification: 94D05, 94D99, 03E70, $03 \mathrm{E} 72$

\section{INTRODUCTION}

FCA (formal concept analysis) was introduced by Wille [11] in 1982, which is an important theory for the research of information structures induced by a binary relation between the set of attributes and objects attributes. The three basic notions of FCA are formal context, formal concept, and concept lattice. A formal context is a kind of information system, which is a tabular form of an object-attribute value relationship [2, 3, 10]. A formal concept is a pair of a set of objects as called the extent and a set of attributes as called the intent.

The concept of soft set was introduced by Molodtsov in 1999 [9], to deal complicated problems and uncertainties. The operations for the soft set theory was introduced by Maji et al. in [4]. Ali et al. [1] proposed new operations modified some concepts introduced by Maji. We have formed a soft context by combining the concepts of the formal context and the soft set defined by the set-valued mapping in [7]. And we introduced and studied the new concepts named soft concepts and soft concepts lattices.

Yao [12] introduced a new concept called an object oriented formal concept in a formal context by using the notion of approximation operations.

We recall that: Let $(U, A, I)$ be a formal context in formal concept analysis, where $U$ is a finite nonempty set of objects, $A$ is a finite nonempty set of attributes and $I$ is a binary relation between $U$ and $A$. For $x \in U$ and $y \in A$, if $(x, y) \in$ $I$, also written as $x I y$. We will denote $x I=\{y \in A \mid x I y\}$; and $I y=\{x \in U \mid x I y\}$.

And, let us consider two set-theoretic operators,

$\square: P(U) \rightarrow P(A): X^{\square}=\{y \in A \mid \forall x \in U(x I y \Rightarrow x \in$ $X)\}$;

$\diamond: P(A) \rightarrow P(U): Y^{\diamond}=\{x \in U \mid \exists y \in A(x I y \wedge y \in Y)\}$.

Then a pair $(X, Y), X \subseteq U, Y \subseteq A$, is called an object oriented formal concept if $X=Y^{\diamond}$ and $Y=X^{\square}$.

Using the facts, we introduced the new notions of objectoriented soft concepts (simply, $m$-concepts) and studied the notion of $m$-concepts and basic properties in [8]. The purpose of this work is to study the topological structure in the family of all object-oriented soft concepts. Furthermore, we introduce the notions of independent $m$-concept and dependent $m$-concept in a soft context. In particular, we show that the set of all independent $m$-concepts completely determines every $m$-concept in a soft context.

\section{PRELIMINARIES}

A formal context is a triplet $(U, A, I)$, where $U$ is a non-empty finite set of objects, $A$ is a nonempty finite set of attributes, and $I$ is a relation between $U$ and $A$. Let $(U, A, I)$ be a formal context. For a pair of elements $x \in U$ and $y \in A$, if $(x, y) \in I$, then it means that object $x$ has attribute $y$ and we write $x I y$. The set of all attributes with a given object $x \in U$ and the set of all objects with a given attribute $y \in A$ are denoted as the following [10,11]:

$$
x^{*}=\{y \in A \mid x I y\} ; y^{*}=\{x \in U \mid x I y\} .
$$

And, the operations for the subsets $X \subseteq U$ and $Y \subseteq A$ are defined as:

$$
X^{*}=\{y \in A \mid \text { for all } x \in X, x I y\} ; \quad Y^{*}=\{x \in
$$
$U \mid$ for all $y \in Y, x I y\}$.

In a formal context $(U, A, I)$, a pair $(X, Y)$ of two sets 
$X \subseteq U$ and $Y \subseteq A$ is called a formal concept of $(U, A, I)$ if $X=Y^{*}$ and $B=Y^{*}$, where $X$ and $Y$ are called the extent and the intent of the formal concept, respectively.

Let $U$ be a universe set and $A$ be a collection of properties of objects in $U$. We will call $A$ the set of parameters with respect to $U$.

A pair $(F, A)$ is called a soft set [9] over $U$ if $F$ is a set-valued mapping of $A$ into the set $P(U)$ of all subsets of the set $U$, i.e.,

$$
F: A \rightarrow P(U) \text {. }
$$

In other words, for $a \in A$, every set $F(a)$ may be considered as the set of $a$-elements of the soft set $(F, A)$.

Let $U=\left\{z_{1}, z_{2}, \ldots, z_{m}\right\}$ be a non-empty finite set of objects, $A=\left\{a_{1}, a_{2}, \ldots, a_{n}\right\}$ a non-empty finite set of attributes, and $F: A \rightarrow P(U)$ a soft set. Then the triple $(U, A, F)$ is called a soft context [7].

And, in a soft context $(U, A, F)$, we introduced the following mappings: For each $Z \in P(U)$ and $Y \in P(A)$,

(1) $\mathbf{F}^{+}: P(A) \rightarrow P(U)$ is a mapping defined as $\mathbf{F}^{+}(Y)=$ $\cap_{y \in Y} F(y)$;

(2) $\mathbf{F}^{-}: P(U) \rightarrow P(A)$ is a mapping defined as $\mathbf{F}^{-}(Z)=$ $\{a \in A: Z \subseteq F(a)\}$;

(3) $\Psi: P(U) \rightarrow P(U)$ is an operation defined as $\Psi(Z)=$ $\mathbf{F}^{+} \mathbf{F}^{-}(Z)$.

Then $Z$ is called a soft concept [7] in $(U, A, F)$ if $\Psi(Z)=$ $\mathbf{F}^{+} \mathbf{F}^{-}(Z)=Z$. The set of all soft concepts is denoted by $s C(U, A, F)$.

In [8], the following operators $\mathbb{F}$ and $\overleftarrow{\mathbb{F}}$ were introduced as follows:

Let $(U, A, F)$ be a soft context. Then for $C \in P(A)$, $X \in P(U)$,

an operator $\mathbb{F}: P(A) \rightarrow P(U)$ is defined by $\mathbb{F}(C)=$ $\cup_{c \in C} F(c)$;

an operator $\overleftarrow{\mathbb{F}}: P(U) \rightarrow P(A)$ is defined by $\overleftarrow{\mathbb{F}}(X)=\{c \in$ $A: F(c) \subseteq X\}$.

Simply, we denote: For $c \in A$ and $x \in U \mathbb{F}(\{c\})=\mathbb{F}(c)$ and $\overleftarrow{\mathbb{F}}(\{x\})=\overleftarrow{\mathbb{F}}(x)$. Obviously, $\mathbb{F}(c)=F(c)$ for $c \in A$.

Theorem 2.1 ([8 )]Let $(U, A, F)$ be a soft context, $S, T \subseteq U$ and $B, C \subseteq A$. Then we have:

(1) If $S \subseteq T$, then $\overleftarrow{\mathbb{F}}(S) \subseteq \overleftarrow{\mathbb{F}}(T)$; if $B \subseteq C$, then $\mathbb{F}(B) \subseteq \mathbb{F}(C)$;

(2) $\mathbb{F} \overleftarrow{\mathbb{F}}(S) \subseteq S ; \overleftarrow{\mathbb{F}} \mathbb{F}(B) \subseteq B$

(3) $\overleftarrow{\mathbb{F}}(S \cap T)=\overleftarrow{\mathbb{F}}(S) \cap \overleftarrow{\mathbb{F}}(T), \mathbb{F}(B \cup C)=\mathbb{F}(B) \cup \mathbb{F}(C)$;

(4) $\overleftarrow{\mathbb{F}}(S)=\overleftarrow{\mathbb{F}} \mathbb{F} \overleftarrow{\mathbb{F}}(S), \mathbb{F}(B)=\mathbb{F} \overleftarrow{\mathbb{F}} \mathbb{F}(B)$

Let us consider an operator defined as follows: For each $X \in P(U)$ in a soft context $(U, A, F)$,

$\mathfrak{F}: P(U) \rightarrow P(U)$ is an operator defined by $\mathfrak{F}(X)=\mathbb{F} \overleftarrow{\mathbb{F}}(X)$
Then $X$ is called an object oriented soft concept (simply, $m$ concept $)[8]$ in $(U, A, F)$ if $\mathfrak{F}(X)=\mathbb{F} \overleftarrow{\mathbb{F}}(X)=X$. The set of all $m$-concepts is denoted by $m(U, A, F)$.

Theorem 2.2 ([8 )]Let $(U, A, F)$ be a soft context. Then we have:

(1) $\mathfrak{F}(X) \subseteq X$ for $X \subseteq U$.

(2) If $X \subseteq Y$, then $\mathfrak{F}(X) \subseteq \mathfrak{F}(Y)$.

(3) $\mathfrak{F}(\mathfrak{F}(X))=\mathfrak{F}(X)$ for $X \subseteq U$.

(4) $\mathfrak{F}(\emptyset)=\emptyset$.

(5) $\mathfrak{F}(X)$ is an $m$-concept.

(6) For $B \subseteq A, \mathbb{F}(B)$ is an m-concept.

(7) For $a \in A, F(a)$ is an m-concept.

(8) $X$ is an $m$-concept if and only if there is some $B \subseteq A$ such that $X=\mathbb{F}(B)$.

\section{MAIN RESULTS}

We assume that a soft set $(F, A)$ is pure [5], that is, $\cup_{a \in A} F(a)=U, \cap_{a \in A} F(a)=\emptyset, F(a) \neq \emptyset$ and $F(a) \neq U$ for each $a \in A$.

Theorem 3.1 Let $(U, A, F)$ be a soft context. Then for $X, Y \in m(U, A, F), \mathfrak{F}(X \cup Y)=\mathfrak{F}(X) \cup \mathfrak{F}(Y)$.

Proof 3.2 Let $X, Y \in m(U, A, F)$. Then by (8) of Theorem 2.2, there are $B, C \subseteq A$ satisfying $\mathbb{F}(B)=X$ and $\mathbb{F}(C)=$ $Y$. Then $X \cup Y=\mathbb{F}(B) \cup \mathbb{F}(C)=\mathbb{F}(B \cup C)$, and so again by Theorem 2.2, $X \cup Y$ is also an $m$-concept. Consequently, $\mathfrak{F}(X \cup Y)=X \cup Y=\mathfrak{F}(X) \cup \mathfrak{F}(Y)$.

Example 3.3 Let $U=\{1,2,3,4,5\}$ and $A=$ $\{a, b, c, d, e, f\}$. Consider a soft context $(U, A, F)$ where a set-valued mapping $F: A \rightarrow P(U)$ is defined by

$$
\begin{gathered}
F(a)=F(d)=\{1,2,4\} ; \quad F(b)=\{2,4,5\} ; \\
F(c)=\{2,4\} ; \quad F(e)=F(f)=\{1,3,5\} .
\end{gathered}
$$

For $X=\{1,2,4\}$ and $Y=\{1,3,5\}, \mathfrak{F}(X \cap Y)=$ $\mathfrak{F}(\{1\})=\emptyset, \mathfrak{F}(X) \cap \mathfrak{F}(Y)=\{1,2,4\} \cap\{1,3,5\}=\{1\}$. So, $\mathfrak{F}(X \cap Y) \neq \mathfrak{F}(X) \cap \mathfrak{F}(Y)$.

From Example 3.2, we know that the family $m(U, A, F)$ is not always a topology on $U$.

A family $\sigma$ of $X$ is called a supra topology [6] on $X$ if $\sigma$ satisfies the conditions: (1) $X, \emptyset \in \sigma$; (2) the union of any number of sets in $\sigma$ belongs to $\sigma$.

Theorem 3.4 ([8 )]Let $(U, A, F)$ be a soft context and $\operatorname{Im}(\mathbb{F})=\{\mathbb{F}(C) \mid \mathbb{F}: P(A) \rightarrow P(U), C \in P(A)\}$. Then

(1) $\operatorname{Im}(\mathbb{F})=m(U, A, F)$ :

(2) For $C_{1}, \cdots, C_{n} \subseteq A, \mathbb{F}\left(C_{1}\right) \cup \mathbb{F}\left(C_{2}\right) \cup \cdots, \mathbb{F}\left(C_{n}\right) \in$ $\operatorname{Im}(\mathbb{F})$. 
Theorem 3.5 Let $(U, A, F)$ be a soft context. Then the family $m(U, A, F)$ is a supra topology on $U$.

Proof 3.6 From Theorem 2.2, it is obtained $U, \emptyset \in$ $m(U, A, F)$. For $X_{1}, \cdots, X_{n} \in m(U, A, F)$, there are $C_{1}, \cdots, C_{n} \subseteq A$ such that $X_{i}=\mathbb{F}\left(C_{i}\right)$. So. $X_{1} \cup \cdots \cup X_{n}=$ $\mathbb{F}\left(C_{1}\right) \cup \cdots \cup \mathbb{F}\left(C_{n}\right) \in \operatorname{Im}(\mathbb{F})=m(U, A, F)$. Consequently, $m(U, A, F)$ is a supra topology on $U$.

Let $(X, \sigma)$ be a supratopological space and $\mathcal{B}$ a family of subsets in $X$. For each supraopen set $G \in \sigma, G$ is a union of any subset of $\mathcal{B}$. Then we will call $\mathcal{B}$ a base for $\sigma$ [6].

Theorem 3.7 For a soft context $(U, A, F)$, the family $\mathcal{F}_{A}=$ $\{F(a) \mid a \in A\}$ is a base for $m(U, A, F)$.

Proof 3.8 Since the soft set $(F, A)$ is pure, $\cup_{a \in A} F(a)=U$. Let $\mathcal{B}=\emptyset \subsetneq \mathcal{F}_{A}$. Then $\cup_{F(a) \in \mathcal{B}} F(a)=\emptyset$.

For any $X \in m(U, A, F)$, from (8) of Theorem 2.2, there is some $B \subseteq A$ such that $X=\mathbb{F}(B)=\cup_{b \in B} F(b)$. So, the family $\mathcal{F}_{A}=\{F(a) \mid a \in A\}$ is a base for $m(U, A, F)$.

Now, to study the property of $\mathcal{F}_{A}=\{F(a) \mid a \in A\}$, we introduce the following concepts:

Definition 3.9 Let $(U, A, F)$ be a soft context. Then for $Z \in m(U, A, F)$,

(1) $Z$ is said to be dependent on $m(U, A, F)$ if there exist $Z_{1}, \cdots, Z_{n} \in m(U, A, F)$ satisfying $Z_{i} \subsetneq Z$ and $Z=\cup Z_{i}$, $i=1, \cdots, n$.

(2) $Z$ is said to be independent of $m(U, A, F)$ if $Z$ is not dependent.

We will denote: $m D=\{Z \in m(U, A, F)$ $Z$ is dependent on $m(U, A, F)\}$;

$Z$ is independent of $m(U, A, F)\}$.

$$
m I=\{Z \in m(U, A, F)
$$

Example 3.10 Let $U=\{1,2,3,4,5\}$ and $A=\{a, b, c, d, e\}$. Consider a soft context $(U, A, F)$, where the set-valued mapping $F: A \rightarrow P(U)$ is defined as follows:

$$
\begin{gathered}
F(a)=\{1,2,4\} ; F(b)=\{1,2,4,5\} ; F(c)=\{2,4\} ; \\
F(d)=\{1,3\} ; F(e)=\{1,5\} .
\end{gathered}
$$

Then,

$m(U, A, F)=\{\emptyset,\{1,3\},\{1,5\},\{2,4\},\{1,2,4\},\{1,3,5\}$, $\{1,2,3,4\},\{1,2,4,5\}, U\}$. For $X=\{1,2,4,5\} \in$ $m(U, A, F)$, we can take two $m$-concepts $Y=F(c)=\{2,4\}$ and $Z=F(e)=\{1,5\}$ in $m(U, A, F)$ satisfying $X \supsetneq Y, Z$ and $X=Y \cup Z$. Hence, $X$ is dependent, while the $m$ concepts $Y, Z$ are independent.

Theorem 3.11 Let $(U, A, F)$ be a soft context. Then

(1) $\emptyset$ and $U$ are dependent.
(2) $m D \cap m I=\emptyset ; \quad m D \cup m I=m(U, A, F)$.

(3) For $Z \in m D$, there is $C \subseteq A$ satisfying for $c \in C$, $F(c) \subsetneq X$ and $\mathbb{F}(C)=Z$.

(4) For $Z \in m I$, there is $c \in A$ satisfying $F(c)=Z$.

Proof 3.12 (1) For the empty set $\emptyset$, there is $\mathcal{B}=\{Z \in$ $m(U, A, F) \mid Z \subsetneq \emptyset\}=\emptyset$. So, $\cup_{Z_{i} \in \emptyset} Z_{i}=\emptyset$.

Now, let $\mathcal{B}=\left\{Z_{i} \in m(U, A, F) \mid Z_{i} \subsetneq U, i=1, \cdots, n\right\}$. Then $\mathcal{B}=m(U, A, F)-\{U\}$. Since the soft set $(F, A)$ is pure, for $a \in A, F(a) \in \mathcal{B}=m(U, A, F)-\{U\}$ and $\cup_{a \in A} F(a)=U$ and so, $U$ is dependent.

(2) It is obvious.

(3) For $Z \in m D$, there are $Z_{1}, \cdots, Z_{n} \in m(U, A, F)$ such that $Z_{i} \subsetneq Z$ and $Z=\cup Z_{i}, i=1, \cdots, n$. From Theorem 2.2, it follows that there are $C_{1}, \cdots, C_{n} \in P(A)$ such that $\mathbb{F}\left(C_{i}\right)=Z_{i}$. Therefore, $\mathbb{F}\left(C_{i}\right) \subsetneq Z$ and $Z=\cup \mathbb{F}\left(C_{i}\right)=$ $\mathbb{F}\left(\cup C_{i}\right), i=1, \cdots, n$. Put $C=\cup_{i=1} C_{i}$. Then $C \subseteq A$ and $\mathbb{F}(C)=Z \supsetneq F(c)$ for $c \in C$.

(4) Let $Z \in m I$. Then there is $C \subseteq A$ such that $\mathbb{F}(C)=Z$. Suppose that for every $c \in C, Z \supsetneq F(c)$, which contradicts to $Z \in m I$. So, there is an element $d \in C$ satisfying $Z=F(d)$.

Theorem 3.13 Let $(U, A, F)$ be a soft context. Then for each $X \in m D$, there is a family $\mathcal{B} \subseteq m I$ satisfying $X=\cup \mathcal{B}$.

Proof 3.14 Let an $m$-concept $X$ be dependent. Suppose $X$ cannot be represented as a union of only elements of $m I$.

Put $\mathcal{S}=\{X \in m D \mid X$ cannot be represented as a union of elements of $m I\}$.

Then, by hypothesis, $\mathcal{S} \neq \emptyset$ and assume that $|\mathcal{S}|=m<$ $|m D|$ where $|m D|$ is the cardinal number of the set $m D$. First, pick up one element $X$ in $\mathcal{S}$ (say, $X_{1}$ ). Then since $X_{1} \in m D$, there is a family $\mathbf{Y}_{\mathbf{1}}=\left\{Y_{11}, \cdots, Y_{1 l}\right\}$ satisfying $Y_{1 i} \in m(U, A, F), Y_{1 i} \subsetneq X_{1}$ and $X_{1}=\cup \mathbf{Y}_{\mathbf{1}}, i=1, \cdots, l$. Additionally, since $X_{1} \in \mathcal{S}, \mathbf{Y}_{\mathbf{1}} \cap \mathcal{S} \neq \emptyset$. Without the loss of generality, we can choose one dependent m-concept in $\mathbf{Y}_{1} \cap \mathcal{S}$, say $X_{2}$. Then $X_{1} \supsetneq X_{2}$, and since $X_{2} \in m D$, there is a family $\mathbf{Y}_{\mathbf{2}}=\left\{Y_{21}, \cdots, Y_{2 m}\right\}$ such that $X_{2} \supsetneq$ $Y_{2 i} \in m(U, A, F)$ and $X_{2}=\cup \mathbf{Y}_{2}, i=1, \cdots, m$. And since $X_{2} \in \mathcal{S}, \mathbf{Y}_{\mathbf{2}} \cap \mathcal{S} \neq \emptyset$.

By repeating this process, finally we can pick up the last element $X_{m}$ in $\mathcal{S}$ that satisfies $X_{1} \supsetneq X_{2} \supsetneq, \cdots \supsetneq X_{n-1} \supsetneq$ $X_{m}$.

Since $X_{m} \in m D$, there is a family $\mathbf{Y}_{\mathbf{m}}=\left\{Y_{m i} \mid Y_{m i} \in\right.$ $m(U, A, F), 1=1, \cdots, r\}$ satisfying $X_{m} \supsetneq Y_{m i}$ and $X_{m}=$ $\cup \mathbf{Y}_{\mathbf{m}}$.

But, since $X_{1} \supsetneq X_{2} \supsetneq, \cdots \supsetneq X_{m}$ and $|\mathcal{S}|=m, \mathcal{S} \cap \mathbf{Y}_{\mathbf{m}}=\emptyset$. So, $X_{m}$ is not in $\mathcal{S}$.

Since $X_{1} \supsetneq X_{2} \supsetneq, \cdots \supsetneq X_{n-1} \supsetneq X_{m}$ and $X_{m}$ is not in $\mathcal{S}$, $X_{m-1}$ is also not in $\mathcal{S}$.

For the same reason as $X_{m-1}, X_{m-2}$ is also not in $\mathcal{S}$. In the end, it leads to $\mathcal{S}=\emptyset$, which is a contradiction. So, every 
International Journal of Engineering Research and Technology. ISSN 0974-3154 Vol.13, No.4 (2020), pp. 660-663

(C) International Research Publication House. https://dx.doi.org/10.37624/IJERT/13.4.2020.660-663

dependent $m$-concept can be represented as a union of only independent $m$-concepts of $m I$.

Theorem 3.15 In a soft context $(U, A, F), m I$ is the smallest base for $m(U, A, F)$.

Proof 3.16 Let $\mathcal{B}$ be a base and $\mathcal{B} \subsetneq m I$. Then for $X \in$ $m I-\mathcal{B}$, there are $S_{1}, \cdots S_{n} \in \mathcal{B}$ such that $X=\cup S_{i}$, which contradicts to $X \in m I$. So, $m I$ is the smallest base.

Theorem 3.17 Let $(U, A, F)$ be a soft context. For $B \subseteq A$, if a set-valued mapping $\varphi: B \rightarrow m I$ defined by $\varphi(b)=F(b)$ for $b \in B$ is surjective, then $\varphi(B)=\{F(b) \mid b \in B\}$ is $a$ base for $m(U, A, F)$.

Proof 3.18 Obvious.

Remark 3.19 Let $(U, A, F)$ be a soft context.

For $m I$,

$$
m(U, A, F)=\{\cup \mathbf{M} \mid \mathbf{M} \subseteq m I\} .
$$

For $\mathcal{F}_{A}=\{F(a) \mid a \in A\}$,

$$
m(U, A, F)=\left\{\cup \mathbf{M} \mid \mathbf{M} \subseteq \mathcal{F}_{A}\right\} .
$$

For $B \subseteq A$ and a surjective mapping $\varphi: B \rightarrow m I$ defined by $\varphi(b)=F(b)$ for $b \in B$,

$$
m(U, A, F)=\{\cup \mathbf{M} \mid \mathbf{M} \subseteq \varphi(B)\} .
$$

For $C \subseteq A$ and a bijective mapping $\psi: C \rightarrow m I$ defined by $\psi(c)=F(c)$ for $c \in B$,

$$
m(U, A, F)=\{\cup \mathbf{M} \mid \mathbf{M} \subseteq \psi(C)\} .
$$

In summary, we have the size relationships for the above bases as follows: For $B, C \subseteq A$,

$$
|m I|=|\psi C| \leq|\varphi B| \leq\left|\mathcal{F}_{A}\right| \leq|m(U, A, F)|
$$

\section{CONCLUSION}

We studied the notion of $m$-dependent and $m$-independent soft concepts in a given soft context. Additionally, we showed that every $m$-dependent soft concept is generated by some $m$ independent soft concepts. In the next study, we will study the various characteristics of such notions and apply these results to object oriented concepts of a formal context.

\section{Acknowledgments}

This research was supported by Basic Science Research Program through the National Research Foundation of Korea(NRF) funded by the Ministry of Education (No. NRF2017R1D1A1B03031399).

\section{REFERENCES}

[1] M. I. Ali, F. Feng, X. Y. Liu, W. K. Min, M. Shabir, On some new operations in soft set theory, Computers and Mathematics with Applications, 57, 2009, 1547-1553.

[2] B. Ganter, R. Wille, Formal Concept Analysis: Mathematical Foundations, Springer, Berlin, 1999.

[3] J. Jin, K. Qin, Z. Pei, Reduction-based approaches towards constructing Galois (concept) lattices, Lecture Notes in Artificial Intelligence, 4062, Springer, Berlin, 2006, 107-113.

[4] P. K. Maji, R. Biswas, A. R. Roy, On soft set theory, Comput. Math. Appl., 45, 2003, 555-562.

[5] Min W. K., Soft sets over a common topological universe, Journal of Intelligent and Fuzzy Systems, 26(5), 2014, 2099-2106.

[6] A. S. Mashhour, A. A. Allam, F. S. Mahmoud, F. H. Khedr, On supra topological spaces, Indian J. Pure and Appl. Math., 14(4), 1983, 502-510.

[7] W. K. Min, Y. K. Kim, Soft concept lattice for formal concept analysis based on soft sets: Theoretical foundations and Applications, Soft Computing, 23(19), 2019, 9657-9668. https://doi.org/10.1007/s00500-018$3532-\mathrm{z}$

[8] W. K. Min, Y. K. Kim, On object-oriented concepts in a soft context defined by a soft set, International Journal of Engineering Research and Technology, 12(11), 2019, 1914-1918.

[9] D. Molodtsov, Soft set theory first results, Computers and Mathematics with Applications, 37, 1999, 19-31.

[10] R. Wille, Concept lattices and conceptual knowledge systems, Computers Mathematics with Applications, 23(6-9), 1992, 493-515.

[11] R. Wille, Restructuring the lattice theory: an approach based on hierarchies of concepts, in: I. Rival (Ed.), Ordered Sets, Reidel, Dordrecht, Boston, 1982, 445470 .

[12] Y. Y. Yao, A comparative study of formal concept analysis and rough set theory in data analysis, RSCTC 2004: Rough Sets and Current Trends in Computing, 2004, 59-68. 